\title{
SYNAPSES ARE LOST DURING AGING IN THE PRIMATE PREFRONTAL CORTEX
}

\author{
A. PETERS, ${ }^{a, b *}$ C. SETHARES ${ }^{a}$ AND J. I. LUEBKE ${ }^{a}$ \\ ${ }^{a}$ Department of Anatomy and Neurobiology, Boston University School \\ of Medicine, 715 Albany Street, Boston, MA 02118-2526, USA \\ ${ }^{b}$ Yerkes National Primate Research Center, Emory University Atlanta, \\ GA 30322, USA
}

\begin{abstract}
An electron microscopic analysis has been carried out on the effects of age on the numerical density of both excitatory (asymmetric) and inhibitory (symmetric) synapses in the neuropil of layers $2 / 3$ and of layer 5 in area 46 from the frontal cortex of behaviorally tested rhesus monkeys. There is no change in the lengths of synaptic junctions with age or in the percentage distribution of synapses relative to the postsynaptic spines and dendritic shafts. However, in layers $2 / 3$ there is an overall loss of about $30 \%$ of synapses from 5 to 30 years of age, and both asymmetric and symmetric synapses are lost at the same rate. In layer 5 the situation is different; the overall loss of synapses is only $20 \%$ and this is almost entirely due to a loss of asymmetric synapses, since there is no significant loss of symmetric synapses from this layer with age. When the synapse data are correlated with the overall cognitive impairment shown by the monkeys, it is found that there is a strong correlation between the numerical density of asymmetric synapses in layers $2 / 3$ and cognitive impairment, with a weaker correlation between symmetric synapse loss and cognitive impairment. In layer 5 on the other hand there is no correlation between synapse loss and cognitive impairment. However synapse loss is not the only factor causing cognitive impairment, since in previous studies of area 46 we have found that age-related alteration in myelin in this frontal area also significantly contributes to cognitive decline. The synapse loss is also considered in light of earlier studies, which show that the frequency of spontaneous excitatory synaptic responses is reduced with age in layers $2 / 3$ neurons. (c) 2008 Published by Elsevier Ltd on behalf of IBRO.
\end{abstract}

Key words: area 46, neuropil, excitatory and inhibitory synapses, electron microscopy.

During normal, non-pathological, aging, there is a marked dysfunction of the primate prefrontal cortex, as shown by a decreased ability to perform cognitive tasks (e.g. Gallagher and Rapp, 1997; Herndon et al., 1997; Moss et al., 1999; Moore et al., 2003) and the question arises as to what is the underlying cause of this cognitive decline. On the basis of early morphological studies it was assumed that the

${ }^{*}$ Correspondence to: A. Peters, Department of Anatomy and Neurobiology, Boston University School of Medicine, 715 Albany Street, Boston, MA 02118-2526, USA. Tel: +1-617-638-4235; fax: +1-617638-4216.

E-mail address: apeters@cajal-1.bu.edu (A. Peters).

Abbreviations: CII, Cognitive Impairment Index; DNMS, delayed nonmatching to sample; DRST, delayed recognition memory span task; PSC, postsynaptic current.

0306-4522/08 $\$ 32.00+0.00$ @ 2008 Published by Elsevier Ltd on behalf of IBRO doi:10.1016/j.neuroscience.2007.07.014 cognitive decline in normal aging is due to neuronal loss, but more recent studies have shown there is not a significant loss of neurons from the neocortex during normal aging (e.g. Peters et al., 1994, 1998a; Peters, 2002; Hof et al., 2000; Peters and Sethares, 2002a). However, this does not mean that neocortical neurons are unaffected by age. Indeed, there is a loss or regression of some of their dendritic branches together with a reduction in the numbers of dendritic spines (e.g. Jacobs et al., 1997; Peters et al., 1998b; Duan et al., 2003). Since dendritic spines are the major recipients of excitatory input to cortical neurons this indicates that there is a loss of input to cortical neurons with age, and such a loss of synapses has been demonstrated in rats (Chen et al., 1995; Wong et al., 1998), monkeys (Uemura, 1980), and humans (Adams, 1987; Masliah et al., 1993), although recently Scheff et al. (2001) concluded that there is no loss of synapses from layers 3 and 5 of human prefrontal cortex.

So far as we are aware, there have been no in-depth analyses of the effects of age on the numerical density of synapses in the neocortex of monkeys, which our group is using as a model of normal aging. The advantage of using rhesus monkeys is that although they can accumulate some senile plaques, they show no signs of Alzheimer's disease, which is always a potentially confounding factor when studies of normal aging are carried out on human subjects. Further, monkeys can be behaviorally tested using many of the cognitive tests used to examine the effects of age on humans (e.g. Moss et al., 1999) and after the behavioral testing has been completed their brains can be adequately preserved for studies designed to determine the morphological basis for any cognitive decline.

We have chosen to examine the effects of age on the numerical density of synapses in the neuropil of area 46 of the rhesus monkey cortex since prefrontal cortex is considered, in part at least, to subserve spatial and reversal learning tasks, as well as recognition memory tasks, and as monkeys age they become impaired on these tasks (Kojima and Goldman-Rakic, 1982; Lai et al., 1995; Fuster 1997; Moss et al., 1997, 2007; Moore et al., 2003). These behavioral changes occur even though there is no change in the volume of prefrontal cortex with age (O'Donnell et al., 1999) and no loss of neurons (Peters et al., 1994; Peters and Sethares, 2002a). However, as we have shown earlier, layer 1 in this cortical area becomes thinner with age, and there is a $30-60 \%$ loss of synapses, a loss that is accompanied by a reduction in the number of apical dendritic branches that occupy this layer (Peters et al., $1998 b)$. Furthermore, this loss of synapses from layer 1 correlates with cognitive decline. 
Although Uemura (1980) showed an overall $20 \%$ loss of synapses from the superior frontal gyrus with age in the rhesus monkey, there is no information about which types of synapses are lost, and whether any loss correlates with cognitive decline. It is important to determine whether changes in the population of synapses with age can explain the increase in the action potential firing rates of layers $2 / 3$ pyramidal cells in area 46 with age (Chang et al., 2005), as well as the accompanying decrease in synaptic excitation and increase in inhibition shown by these neurons (Luebke et al., 2004).

\section{Estimating numerical density of synapses with age}

There are two main methods for estimating the numerical density of synapses using thin sections of tissue prepared for electron microscopy; these are the size-frequency method (Colonnier and Beaulieu, 1985) and the disector method (e.g. Sterio, 1984; Calverley et al., 1988). In comparisons of the data generated by these two methods, both DeFelipe et al. (1999) and we (Peters et al., 2001) have shown that the methods give comparable results, and that when the effect of age on the numerical density of a population of synapses is being estimated, both methods reveal the same trends in changes with age (Peters et al., 2001). This being so, we have chosen to use the sizefrequency method to make our estimations. This method is more efficient and much easier to apply than the disector method, which involves preparing serial thin sections and examining identical fields in nearby sections of a series.

\section{EXPERIMENTAL PROCEDURES}

\section{Tissue specimens and processing}

The ages and sexes of the 17 rhesus monkeys (Macaca mulatta) used in the study are given in Tables 2 and 3 . Five of the monkeys were young (5-10 years of age), six were middle aged (11-20 years of age), and six were old (over 20 years of age). Details of how the brains of these monkeys were fixed for morphological examination are given in Peters et al. (1994). Briefly, the monkey was pre-anesthetized with ketamine $(0.5 \mathrm{mg} / \mathrm{kg})$ after which sodium barbital was administered i.v. (15 mg/kg to effect) until the monkey was deeply anesthetized and a state of areflexia achieved. The monkey was then intubated and artificially respired with a mixture of $5 \% \mathrm{CO}_{2}$ and $95 \% \mathrm{O}_{2}$. The chest was opened and the monkey perfused intracardially with a warm solution of $1 \%$ paraformaldehyde and $1.25 \%$ glutaraldehyde in a $0.1 \mathrm{M}$ cacodylate or phosphate buffer at $\mathrm{pH}$ 7.4. The brain was then removed and one hemisphere stored in a cold solution of $2 \%$ paraformaldehyde and $2.5 \%$ glutaraldehyde in the same $0.1 \mathrm{M}$ buffer used for the perfusion. The perfusions were carried out in full accordance with the approved Institutional Animal Care and Use Committee Regulations, and in accordance with the NIH Publication Guide for the Care and Use of Laboratory Animals. All efforts were made to minimize the number of animals used and their suffering.

Several pieces of cortex were removed from the lower bank inside the sulcus principalis of each monkey, at the level of the rostrum of the corpus callosum. This portion of the prefrontal cortex is part of area 46 according to the designation given by Walker (1940), and was chosen because it has a relatively even thickness (Peters et al., 1994). The pieces of cortex were then osmicated, dehydrated, stained en block with uranyl acetate and embedded in araldite.

\section{Preparation of sections}

The plastic-embedded blocks of area 46 used for this study were sectioned in a plane at right angles to the pial surface of the cortex, and oriented so that long lengths of the apical dendrites of pyramidal cells were evident in the $1 \mu \mathrm{m}$ thick sections that were stained with Toluidine Blue for an assessment of the adequacy of the orientation of the tissue block face and the quality of the preservation. Thin sections were then cut from one block of tissue, mounted on copper mesh grids, and stained with lead citrate.

The thin sections were first examined at low magnification in the electron microscope and a drawing made of the outline of the section relative to the bars of the copper mesh grid. The boundary between layer 1 and layer 2 was also marked, as well as the locations of the white/gray matter border and the border between layers 5 and $6 \mathrm{~A}$. Next the location of layer 4 , which is of somewhat variable thickness and composed of small neurons, was determined and its position and thickness were marked on the drawing. In a systematic fashion two sets of micrographs of the neuropil were then taken at a magnification of $\times 6000$. One set of micrographs was of the neuropil in layers $2 / 3$ and the other of the neuropil in layer 5 , and each set consisted of at least 10 micrographs per monkey. Micrographs were not taken in layer 4, because this layer is thin and of variable thickness, making it difficult to carry out a systematic analysis of synapse densities. Care was taken to avoid including the cell bodies of neurons and neuroglia, and profiles of large dendrites and of blood vessels in the micrographs. The negatives were then enlarged and printed at a final magnification of $\times 12,500$. In one monkey, AM 178, the fixation of layer 5 was not good enough to make synapse counts reliable and so no data are available for layer 5 in this monkey.

\section{Counting synapses}

The size frequency method uses the empirical formula suggested by Colonnier and Beaulieu (1985). The formula is $N_{V}=N_{A} / d$, where $N_{V}$ is the number of synapses per unit volume, $N_{A}$ is the number of synaptic junctions per unit area of an electron micrograph, and $d$ is the mean length of densities associated with the synaptic junctions. The profiles of synapses were marked on each micrograph of a set: a synapse was only marked if the synaptic junction was apparent, and if at least two synaptic vesicles were seen in the presynaptic component of the synapse. The synapses were marked as being of the asymmetric or symmetric type, based on the prominence of the postsynaptic junction and the morphology of the synaptic vesicles (Colonnier, 1968), as will be described in more detail in the Results section. In general between 250-350 asymmetric synapses and 30-50 symmetric synapses were analyzed in each set of micrographs.

Next, the lengths of densities of the synaptic junctions were measured using a $\times 10$ magnifying lens with a graticule calibrated in $0.1 \mathrm{~mm}$ increments. If a synaptic junction profile was curved, the length of the junction was measured between its two ends. For each set of prints the lengths of the synaptic densities of at least 100 asymmetric and all of the symmetric synapses, usually at least 50 , were measured, and the mean lengths of the postsynaptic densities calculated. When the mean junctional lengths $(d)$ had been calculated, and the mean number of synaptic profiles per unit area $\left(N_{A}\right)$ had been determined, the values were entered into the formula of Colonnier and Beaulieu (1985) to estimate a value for $N_{v}$.

Given that each print in a set generates a single count $\left(N_{A}\right)$, the coefficient of error for each set of prints was determined, and it was assumed that a sufficient number of counts had been made when the coefficient of error was $10 \%$ or less. If the coefficient of error did not reach that criterion, additional micrographs were used to make additional counts, until the acceptable $10 \%$ coefficient of error was attained. 
Table 1. Percentage distribution of synapses in the neuropil

\begin{tabular}{|c|c|c|c|c|c|c|}
\hline \multirow[t]{2}{*}{ Layer } & \multicolumn{2}{|c|}{ Asymmetric synapses (\%) } & \multirow{2}{*}{$\begin{array}{l}\text { No. of synapses } \\
\text { examined }\end{array}$} & \multicolumn{2}{|c|}{ Symmetric synapses (\%) } & \multirow{2}{*}{$\begin{array}{l}\text { No. of synapses } \\
\text { examined }\end{array}$} \\
\hline & Axospinous & Axodendritic & & Axospinous & Axodendritic & \\
\hline \multicolumn{7}{|l|}{ Layer $2 / 3$} \\
\hline Young & $83.5 \pm 5.0$ & $16.5 \pm 5.0$ & 1767 & $21.5 \pm 3.9$ & $78.5 \pm 3.9$ & 205 \\
\hline Old & $77.0 \pm 2.2$ & $23.0 \pm 2.2$ & 1197 & $28.0 \pm 6.7$ & $72.0 \pm 6.7$ & 157 \\
\hline \multicolumn{7}{|l|}{ Layer 5} \\
\hline Young & $77.8 \pm 2.6$ & $23.3 \pm 2.6$ & 1082 & $25.8 \pm 5.7$ & $74.2 \pm 5.7$ & 149 \\
\hline Old & $75.0 \pm 4.0$ & $25.0 \pm 4.0$ & 922 & $23.0 \pm 6.0$ & $77.0 \pm 5.7$ & 124 \\
\hline
\end{tabular}

\section{Assessment of the postsynaptic components of asymmetric and symmetric synapses}

To determine which dendritic components receive asymmetric and symmetric synapses in layers $2 / 3$ and in layer 5 , the sets of electron micrographs of those layers taken from the neuropil of four young (AM 76; AM 129; AM 47; and AM 53) and four old (AM 12; AM 62; AM 26; and AM 41) monkeys were examined, and the synapses assessed as being either axospinous or axodendritic. Only one or two axo-axonal synapses were encountered in the neuropil and they were not included in the analysis. The data derived from the examination of the four young monkeys were then pooled, as were the data from the four old monkeys, to provide an assessment of the distribution of synapses relative to the dendritic shafts and spines and to ascertain if there is a change in the percentage distribution of synapses on these components with age. The data are presented in Table 1.

\section{Behavioral testing}

With the exception of one 5-year-old monkey (AM 16), all of the monkeys used in this study had been behaviorally tested. The behavioral tests used to assess the cognitive status of these monkeys have been described in previous publications (e.g. Herndon et al., 1997; Moss et al., 1999, 2007; Peters et al., 1996, 2000; Killiany et al., 2000). The assessment is made on the basis of the scores obtained by the monkeys on three visual recognition tasks. These tasks are the delayed nonmatching to sample (DNMS) task, a DNMS task with a 2 min delay, and the spatial delayed recognition memory span task (DRST). Significant impairment on a given behavioral task is defined as $>200$ errors for the DNMS basic task; $<78 \%$ correct for the DNMS 2 min delay task; and a span of $<2.5$ for the spatial DRST task (Herndon et al., 1997). From the scores obtained on these tasks an overall measure of cognitive impairment, the Cognitive Impairment Index (CII) is derived (Peters et al., 2001). Essentially, the higher the Cll score, the more a monkey is impaired. In general monkeys with CII scores lower than 1.5 are considered to be non-impaired, ones with $\mathrm{Cll}$ scores between 1.5 and 2.5 are considered to be mildly impaired, and monkeys with scores over 2.5 are considered to be severely impaired. The behavioral scores obtained by the monkeys in this study are given in Table 4.

\section{Statistical analyses}

A series of statistical analyses was performed to examine the relationships between age and synapse numbers, as well as the behavioral variables. In our analyses of age as an independent factor we also employed piecewise linear models to account for the possibility of non-linearity between synapse numbers and age. This allowed us to estimate separate slopes for three age intervals in the data in a comprehensive model. The age intervals were those less than 10 years of age (young); those from 10 to 20 years of age (middle aged); and those older than 20 years (old). These nodal points were chosen prior to conducting the analyses and were based on theoretical grounds.

\section{RESULTS}

\section{Morphology of synapses}

As pointed out, morphologically there are two types of synapses in the cerebral cortex. When tissue is fixed using aldehydes these two types are referred to as asymmetric and symmetric synapses (Colonnier, 1968), and it is generally agreed that asymmetric synapses are excitatory and that symmetric synapses are inhibitory in function (e.g. see Peters and Palay, 1996). Distinguishing between these two synaptic types morphologically depends upon the width of the synaptic junctions, the thickness of the postsynaptic densities, and the shapes of the synaptic vesicles.

Asymmetric synapses have uniformly round synaptic vesicles in the axon terminal, a wide cleft between the preand postsynaptic membranes, and a prominent postsynaptic density. Reconstructions from serial thin sections through asymmetric synapses show that viewed en face a postsynaptic density can have a rather irregular shape and that the largest densities can have one or two perforations (Peters and Kaiserman-Abramof, 1969). Consequently in thin sections the postsynaptic density can occupy almost the entire postsynaptic membrane (e.g. Fig. $1 a ; A_{3}$ and $d_{3}$ ), or only a portion of it (e.g. Fig. $1 \mathrm{a}: \mathrm{A}_{1}$ and $\mathrm{sp}_{1}$; arrow), while in sections through a perforation the density can appear as two separate parts (e.g. Fig. $1 \mathrm{a} ; \mathrm{A}_{2}$ and $\mathrm{sp}_{2}$; arrows). In general asymmetric synapses involving dendritic shafts have unperforated postsynaptic densities (Fig. 1a; $A_{3}$ and $d_{3}$ : Fig. $1 b ; A_{1}$ and $d_{1}$ ).

In contrast to the asymmetric ones, symmetric synapses involving both dendritic spines (Fig. 1a; $\mathrm{S}_{2}$ and $\mathrm{sp}_{2}$ ) and dendritic shafts (Fig. $1 b ; S_{1}$ and $d_{1}$ ) have a thinner and less prominent postsynaptic density, a narrower synaptic cleft, and pleomorphic synaptic vesicles, so that some profiles of vesicles are round and others are elongate. With the exception of a few axo-axonic synapses involving axon initial segments, all synapses in the neuropil are either axodendritic or axospinous.

As shown in Fig. 1, in the neuropil of the cerebral cortex most asymmetric synapses involve dendritic spines, the profiles of which are generally flattened on the face 


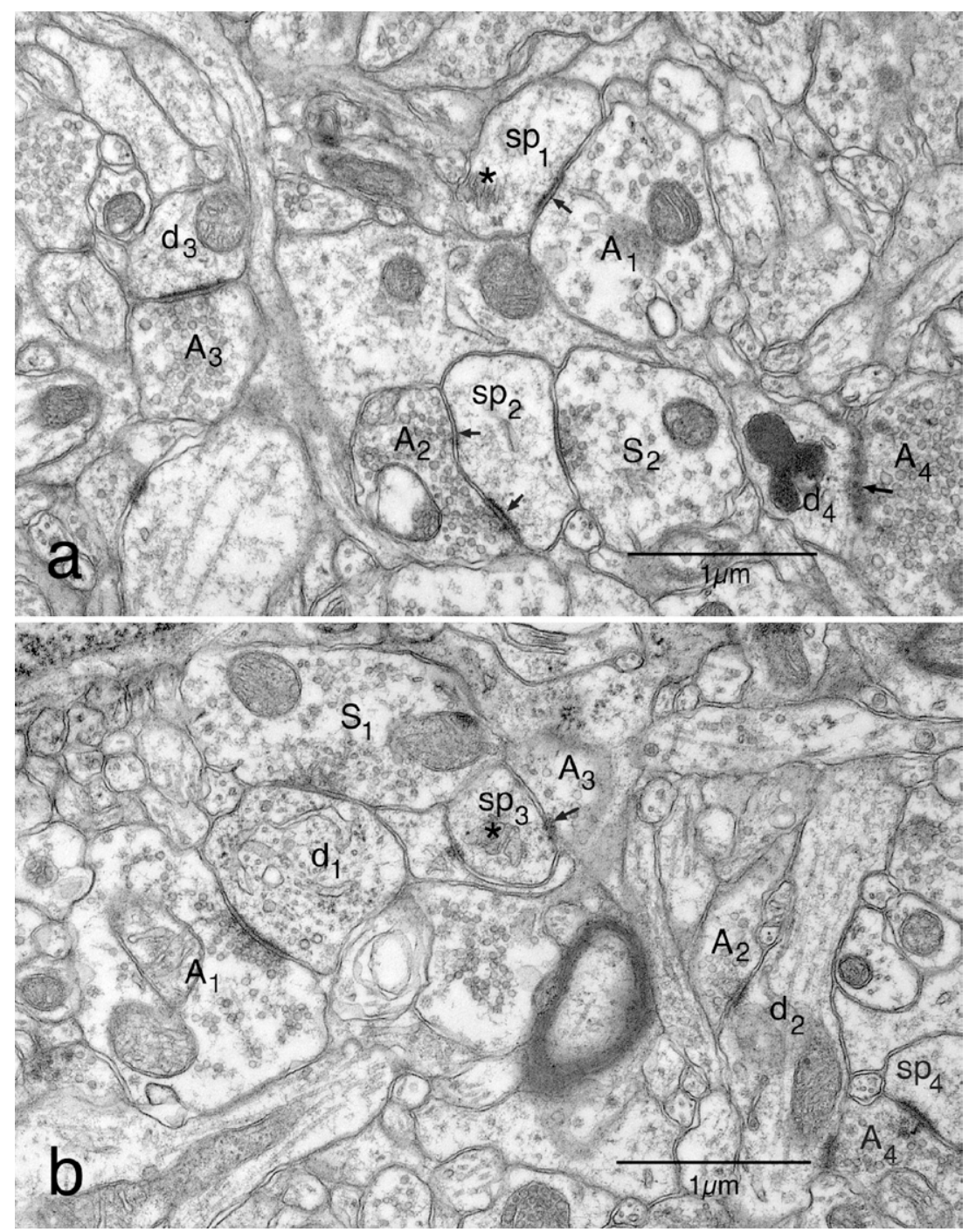

Fig. 1. Electron micrographs of the neuropil in layer 5 of area 46 to show the types of synapses present. For a more complete description of these micrographs see the Results section. (a) The field contains two dendritic spines. One of the spines $\left(\mathrm{sp}_{1}\right)$ is forming an asymmetric synapse with an axon terminal $\left(A_{1}\right)$ containing round synaptic vesicles. The other spine $\left(s_{2}\right)$ is synapsing with one axon terminal $\left(A_{2}\right)$ forming an asymmetric synapse and a second axon terminal $\left(S_{2}\right)$ with pleomorphic vesicles forming a symmetric synapse. Also in this field are a dendrite $\left(d_{3}\right)$ forming an asymmetric synapse with an axon terminal $\left(A_{3}\right)$ containing round vesicles, and a dendrite $\left(d_{4}\right)$ forming an asymmetric synapse with a terminal $\left(A_{4}\right)$ containing round vesicles. The junction (arrow) between the two components of this synapse is sectioned obliquely. (b) On the left of this field is a transversely sectioned dendrite $\left(d_{1}\right)$ forming an asymmetric synapse with an axon terminal $\left(A_{1}\right)$ containing round synaptic vesicles, and a symmetric synapse with an axon terminal $\left(S_{1}\right)$ containing pleomorphic vesicles. Another axon terminal $\left(A_{2}\right)$ is forming an asymmetric synapse with the shaft of a second dendrite $\left(d_{2}\right)$. Also present in the field is a dendritic spine $\left(\mathrm{sp}_{3}\right)$ forming an asymmetric synapse with an axon terminal $\left(\mathrm{A}_{3}\right)$. The axon terminal and the synaptic junction (arrow) are both sectioned obliquely. There is also a second asymmetric axospinous synapse between an axon terminal $\left(\mathrm{A}_{4}\right)$ a dendritic spine $\left(\mathrm{sp}_{4}\right)$ in the field. Terminal $\left(\mathrm{A}_{4}\right)$ also synapses with dendrite $\left(\mathrm{d}_{2}\right)$.

involving the synapse. The profiles of dendritic spines can be recognized by the presence of flocculent material in the cytoplasm, which may also contain a spine apparatus (Fig. 1; asterisks). Each dendritic spine forms only one asymmetric synapse. Other asymmetric synapses are axodendritic, and the profiles of dendrites (Fig. $1 b ; d_{1}$ and $d_{2}$ ) can be identified by the presence of microtubules in the cytoplasm, which sometimes contains mitochondria (Fig. 1a; $d_{3}$ : Fig. 1b; $d_{2}$ ). Symmetric synapses can also involve dendrites and dendritic spines, and when dendritic spines are seen to form two synapses, one of the synapses is invariably asymmetric and the other one symmetric (Fig. $1 \mathrm{a} ; \mathrm{sp}_{2}$ ).
While most synapses can be readily recognized as to their type, some problems can arise when synapses are sectioned obliquely. However, a diagnosis as to synaptic type can almost always be made on the basis of the features that are visible. For example, in Fig. $1 \mathrm{~b}$ the apposition between dendritic spine $\mathrm{sp}_{3}$ and terminal $A_{3}$ is obviously an asymmetric axospinous synapse since the postsynaptic component contains a spine apparatus (asterisk), a small portion of the junction is visible (arrow), and the glancing section through the axon terminal $\left(A_{2}\right)$ shows that it contains round synaptic vesicles. Another example is shown in the bottom right of Fig. 1a, where a dendritic 
shaft $\left(d_{4}\right)$ is synapsing with an axon terminal that contains round synaptic vesicles $\left(A_{4}\right)$, In this case the synaptic junction (arrow), is sectioned obliquely, but it is clearly visible and so this synapse can be classified as an asymmetric axo-dendritic synapse. Axon terminal $A_{4}$ is also synapsing with dendrite $\mathrm{d}_{2}$.

In the rare cases in which the plane of section passes more or less parallel to the synaptic junction, the profile is only counted as a synapse if the section passes through the synaptic density and not through the presynaptic grid, which can be recognized by the hexagonally arranged grid of presynaptic densities (see Peters et al., 1991).

It should be pointed out that the appearance of synapses does not alter with age and so this description applies equally well to synapses in young, middle aged, and old monkeys.

\section{Postsynaptic components of synapses}

As shown in Table 1, in layers $2 / 3$ of young monkeys $83.5 \%$ of asymmetric synapses involve dendritic spines, and only $16.5 \%$ involve the shafts of dendrites. For symmetric synapses, the converse obtains, since $78.5 \%$ of these synapses involve the shafts of dendrites and only $21.5 \%$ involve dendritic spines. Virtually the same distribution of synapses also obtains for layer 5 neuropil. The distribution of synapses is virtually the same in old monkeys, and an analysis of the data for young and old monkeys, using a two-tailed $t$-test, showed that the distribution of the postsynaptic components receiving the two types of synapses does not alter significantly with age.

\section{Numerical density of synapses}

As shown in Tables 2 and 3, and in Figs. 2 and 3, there is an age-related loss of synapses from both layers $2 / 3$ and layer 5 .
In layers $2 / 3$ the lengths of the profiles of asymmetric synaptic junctions do not change with age $(P=0.504)$, and neither does the mean length for symmetric synaptic junctions $(P=0.55)$. The overall mean length of asymmetric synapses is $0.35 \pm 0.02 \mu \mathrm{m}$, while the symmetric synapses junctions are shorter, having an overall mean length of $0.29 \pm 0.01 \mu \mathrm{m}$ (Table 2).

In young monkeys the mean numerical density for all synapses is $595 \times 10^{6}$ per $\mathrm{mm}^{3}$, while for old monkeys it is $435 \times 10^{6}$ per $\mathrm{mm}^{3}$. This overall loss of synapses correlates significantly with age $(P=<0.0001)$ and it represents an overall loss of about $30 \%$ of synapses. Asymmetric synapses account for some $87 \%$ of all synapses in layers $2 / 3$, and the mean numerical density of asymmetric synapses in young monkeys is $520 \times 10^{6}$ per $\mathrm{mm}^{3}$ and for old monkeys it is $374 \times 10^{6}$ per $\mathrm{mm}^{3}$. An overall linear analysis (Fig. 2) shows that the loss of asymmetric synapses correlates significantly with age $(P=0.0001)$, and interestingly, a piecewise analysis (Fig. 2; dashed line) shows that most of the loss of these synapses occurs after 20 years of age $(P=0.0021)$.

Symmetric synapses account for some $13 \%$ of all synapses, while the mean numerical density of symmetric synapses is $74 \times 10^{6}$ per $\mathrm{mm}^{3}$ in young monkeys and $58 \times 10^{6}$ per $\mathrm{mm}^{3}$ in old monkeys (Fig. 2). An overall linear analysis of the data shows that there is there is a correlation between loss of symmetric synapses and age $(P=0.0164)$, and a piecewise analysis (not shown) indicates that the loss occurs gradually at all ages.

When the ratios of the numerical densities for asymmetric versus symmetric synapses are plotted against age (Fig. 4) there is no significant difference $(P=0.287)$ in the ratio with age, suggesting that both types of synapses are being lost at the same rate.

Table 2. Synapses in layers $2 / 3$

\begin{tabular}{|c|c|c|c|c|c|c|c|c|c|}
\hline \multirow{2}{*}{$\begin{array}{l}\text { Animal } \\
\text { AM no. }\end{array}$} & \multirow[t]{2}{*}{ Age $(y)$} & \multirow[t]{2}{*}{ Sex } & \multicolumn{3}{|l|}{ Asymmetric } & \multicolumn{3}{|l|}{ Symmetric } & \multirow{2}{*}{$\begin{array}{l}\text { Total Nv } \\
10^{6} / \mathrm{mm}^{3}\end{array}$} \\
\hline & & & $\begin{array}{l}\text { Mean length of } \\
\text { junctions }(\mu \mathrm{m})\end{array}$ & $\mathrm{Nv} \times 10^{6} / \mathrm{mm}^{3}$ & S.D. & $\begin{array}{l}\text { Mean length of } \\
\text { junctions }(\mu \mathrm{m})\end{array}$ & $\mathrm{Nv} \times 10^{6} / \mathrm{mm}^{3}$ & S.D. & \\
\hline 16 & 5 & M & 0.35 & 543 & 70 & 0.30 & 61 & 22 & 605 \\
\hline 76 & 6 & $\mathrm{~F}$ & 0.33 & 542 & 89 & 0.28 & 70 & 21 & 613 \\
\hline 129 & 7 & $\mathrm{~F}$ & 0.35 & 541 & 85 & 0.28 & 83 & 21 & 625 \\
\hline 47 & 9 & $M$ & 0.33 & 485 & 85 & 0.27 & 82 & 10 & 567 \\
\hline 53 & 10 & $\mathrm{M}$ & 0.37 & 490 & 63 & 0.30 & 76 & 16 & 561 \\
\hline Means & & & 0.35 & 520 & 78 & 0.29 & 74 & 18 & 595 \\
\hline 42 & 12 & $M$ & 0.37 & 445 & 47 & 0.28 & 70 & 19 & 515 \\
\hline 144 & 15 & $M$ & 0.32 & 479 & 63 & 0.30 & 56 & 5 & 535 \\
\hline 143 & 16 & $M$ & 0.33 & 468 & 43 & 0.29 & 63 & 22 & 531 \\
\hline 221 & 18 & $\mathrm{~F}$ & 0.33 & 560 & 51 & 0.28 & 78 & 8 & 638 \\
\hline 209 & 19 & $M$ & 0.32 & 491 & 51 & 0.29 & 63 & 13 & 555 \\
\hline 133 & 19 & $M$ & 0.32 & 507 & 11 & 0.30 & 64 & 21 & 570 \\
\hline Means & & & 0.33 & 491 & 44 & 0.29 & 67 & 15 & 557 \\
\hline 178 & 22 & $\mathrm{~F}$ & 0.37 & 347 & 41 & 0.30 & 51 & 5 & 416 \\
\hline 19 & 25 & $\mathrm{~F}$ & 0.36 & 440 & 29 & 0.29 & 63 & 15 & 503 \\
\hline 12 & 27 & $\mathrm{~F}$ & 0.35 & 407 & 74 & 0.27 & 78 & 14 & 485 \\
\hline 62 & 27 & $M$ & 0.38 & 343 & 77 & 0.31 & 51 & 23 & 394 \\
\hline 26 & 29 & $\mathrm{~F}$ & 0.38 & 385 & 61 & 0.31 & 58 & 19 & 443 \\
\hline 41 & 32 & $\mathrm{~F}$ & 0.34 & 320 & 38 & 0.31 & 46 & 12 & 366 \\
\hline Means & & & 0.36 & 374 & 53 & 0.30 & 58 & 15 & 435 \\
\hline
\end{tabular}


Table 3. Synapses in layer 5

\begin{tabular}{|c|c|c|c|c|c|c|c|c|c|}
\hline \multirow{2}{*}{$\begin{array}{l}\text { Animal } \\
\text { AM no. }\end{array}$} & \multirow[t]{2}{*}{ Age $(y)$} & \multirow[t]{2}{*}{ Sex } & \multicolumn{3}{|l|}{ Asymmetric } & \multicolumn{3}{|l|}{ Symmetric } & \multirow{2}{*}{$\begin{array}{l}\text { Total Nv } \\
10^{6} / \mathrm{mm}^{3}\end{array}$} \\
\hline & & & $\begin{array}{l}\text { Mean length of } \\
\text { junction }(\mu \mathrm{m})\end{array}$ & $\mathrm{Nv} \times 10^{6} / \mathrm{mm}^{3}$ & S.D. & $\begin{array}{l}\text { Mean length of } \\
\text { junction }(\mu \mathrm{m})\end{array}$ & $\mathrm{Nv} \times 10^{6} / \mathrm{mm}^{3}$ & S.D. & \\
\hline 16 & 5 & $M$ & 0.36 & 350 & 35 & 0.27 & 48 & 10 & 398 \\
\hline 76 & 6 & $\mathrm{~F}$ & 0.34 & 407 & 96 & 0.26 & 69 & 19 & 475 \\
\hline 129 & 7 & $\mathrm{~F}$ & 0.35 & 410 & 63 & 0.31 & 58 & 14 & 468 \\
\hline 47 & 9 & $M$ & 0.35 & 290 & 76 & 0.28 & 50 & 19 & 340 \\
\hline 53 & 10 & $M$ & 0.34 & 427 & 43 & 0.31 & 61 & 12 & 489 \\
\hline Means & & & 0.35 & 377 & 63 & 0.29 & 57 & 15 & 434 \\
\hline 42 & 12 & $M$ & 0.38 & 330 & 108 & 0.28 & 56 & 21 & 386 \\
\hline 144 & 15 & $M$ & 0.33 & 329 & 81 & 0.30 & 47 & 13 & 376 \\
\hline 143 & 16 & $M$ & 0.37 & 372 & 70 & 0.31 & 49 & 11 & 421 \\
\hline 221 & 18 & $\mathrm{~F}$ & 0.38 & 378 & 53 & 0.28 & 62 & 21 & 440 \\
\hline 209 & 19 & $M$ & 0.35 & 395 & 53 & 0.28 & 58 & 21 & 453 \\
\hline 133 & 19 & $M$ & 0.31 & 341 & 80 & 0.27 & 52 & 20 & 393 \\
\hline Means & & & 0.35 & 358 & 74 & 0.29 & 54 & 18 & 412 \\
\hline 178 & 22 & $\mathrm{~F}$ & & & & & & & \\
\hline 19 & 25 & $\mathrm{~F}$ & 0.40 & 262 & 34 & 0.33 & 49 & 14 & 311 \\
\hline 12 & 27 & $\mathrm{~F}$ & 0.37 & 328 & 57 & 0.30 & 56 & 21 & 384 \\
\hline 62 & 27 & $M$ & 0.37 & 315 & 53 & 0.29 & 48 & 15 & 363 \\
\hline 26 & 29 & $\mathrm{~F}$ & 0.38 & 294 & 51 & 0.30 & 60 & 17 & 353 \\
\hline 41 & 32 & $\mathrm{~F}$ & 0.34 & 302 & 53 & 0.27 & 48 & 10 & 350 \\
\hline Means & & & 0.37 & 300 & 50 & 0.30 & 52 & 15 & 352 \\
\hline
\end{tabular}

In layer 5 there is also no change in the lengths of profiles of asymmetric $(P=0.284)$ and of symmetric $(P=0.474)$ synaptic junctions with age (Table 3 ). The mean length of the asymmetric synapse junctions is $0.36+0.02 \mu \mathrm{m}$, which is slightly longer than in layers $2 / 3$, but the mean length of symmetric synaptic junctions is the same as in layers $2 / 3$, namely $0.29+0.1 \mu \mathrm{m}$. As in layers $2 / 3$, about $87 \%$ of the synapses in young monkeys are asymmetric ones.

The numerical density of synapses in layer 5 of young monkeys is about $30 \%$ less than in layers $2 / 3$. Thus in layer

Layer 2/3: Synapses v. Age

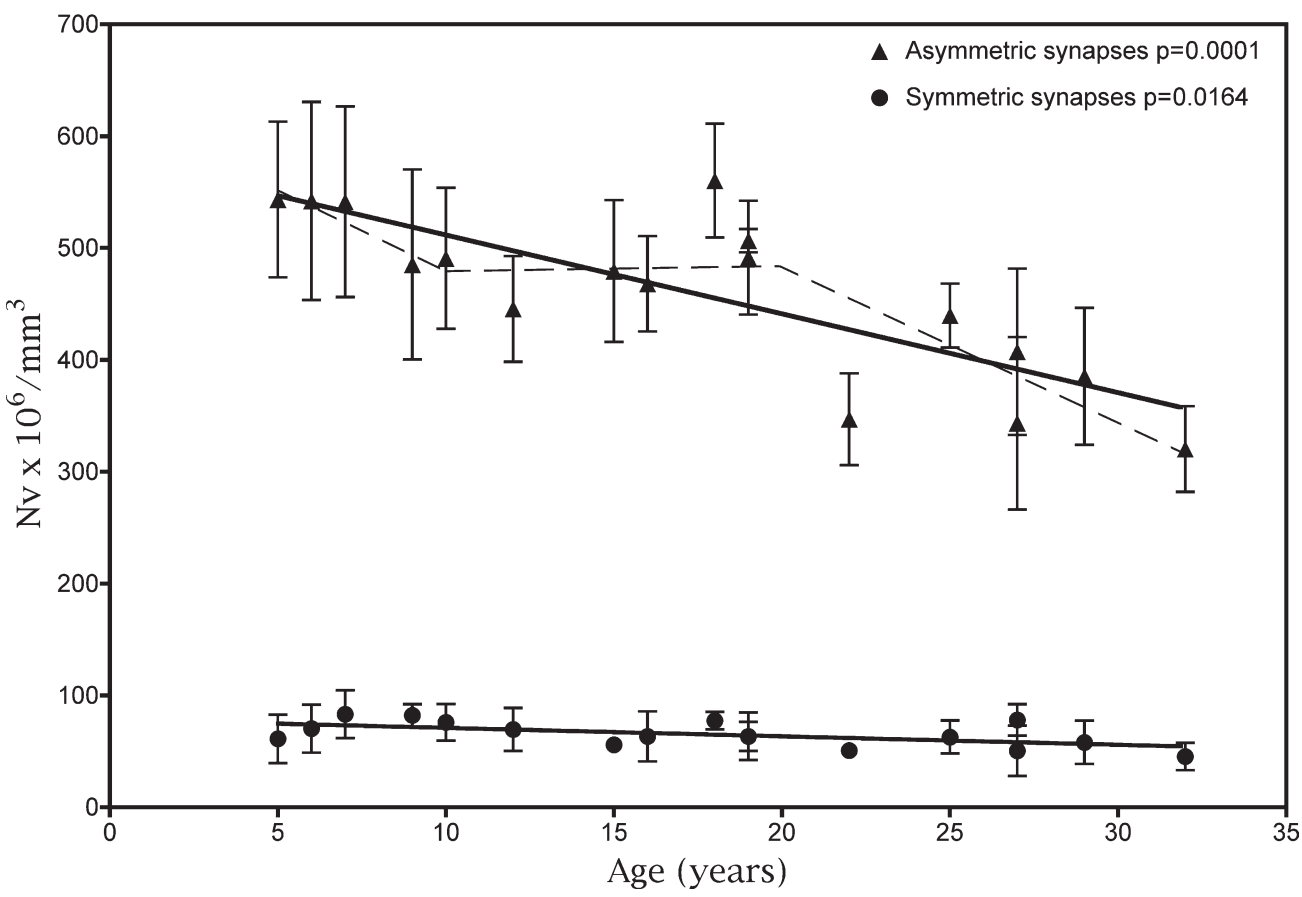

Fig. 2. A plot of the number of asymmetric and symmetric synapses present per $\mathrm{mm}^{3}$ in the neuropil of layers $2 / 3$ against age. The dashed line superimposed on the plot for asymmetric synapses is the piecewise analysis, which shows that most of the loss of these synapses occurs after 20 years of age. 


\section{Layer 5: Synapses v. Age}

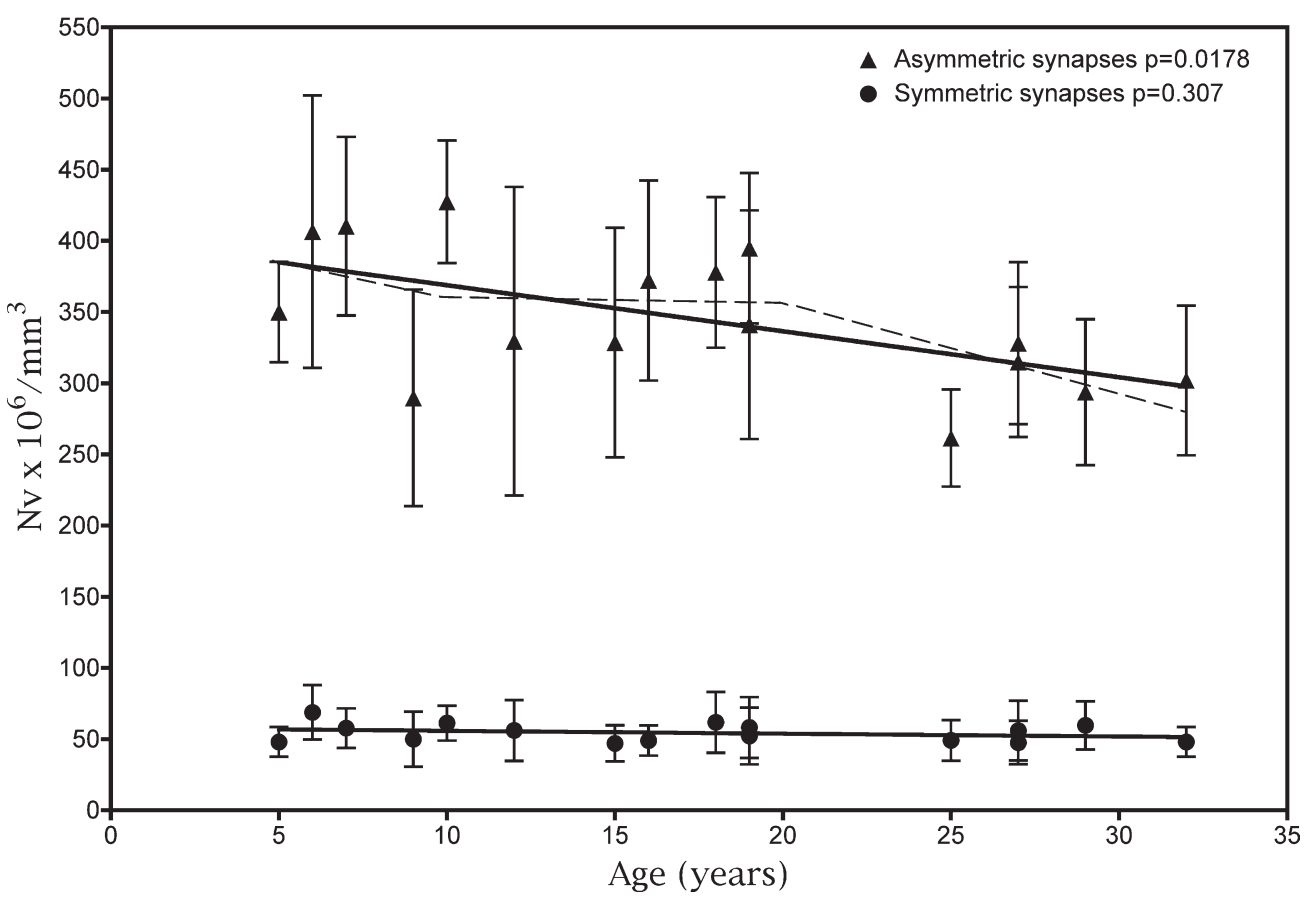

Fig. 3. A plot of the number of asymmetric and symmetric synapses present per $\mathrm{mm}^{3}$ in the neuropil of layer 5 against age. The dashed line superimposed on the plot for asymmetric synapses is the piecewise analysis, which shows that the loss of these synapses with age is gradual.

5 the overall synaptic density in the neuropil of young monkeys is $434 \times 10^{6}$ per $\mathrm{mm}^{3}$ (Table 3 ), and in old monkeys the overall synaptic density in layer 5 is $352 \times 10^{6}$ per $\mathrm{mm}^{3}$. Consequently the overall loss of synapses from layer 5 with age is about $20 \%$ : somewhat less than in layers $2 / 3$, from which the overall loss is about $30 \%$.

Asymmetric/Symmetric synapses v. Age

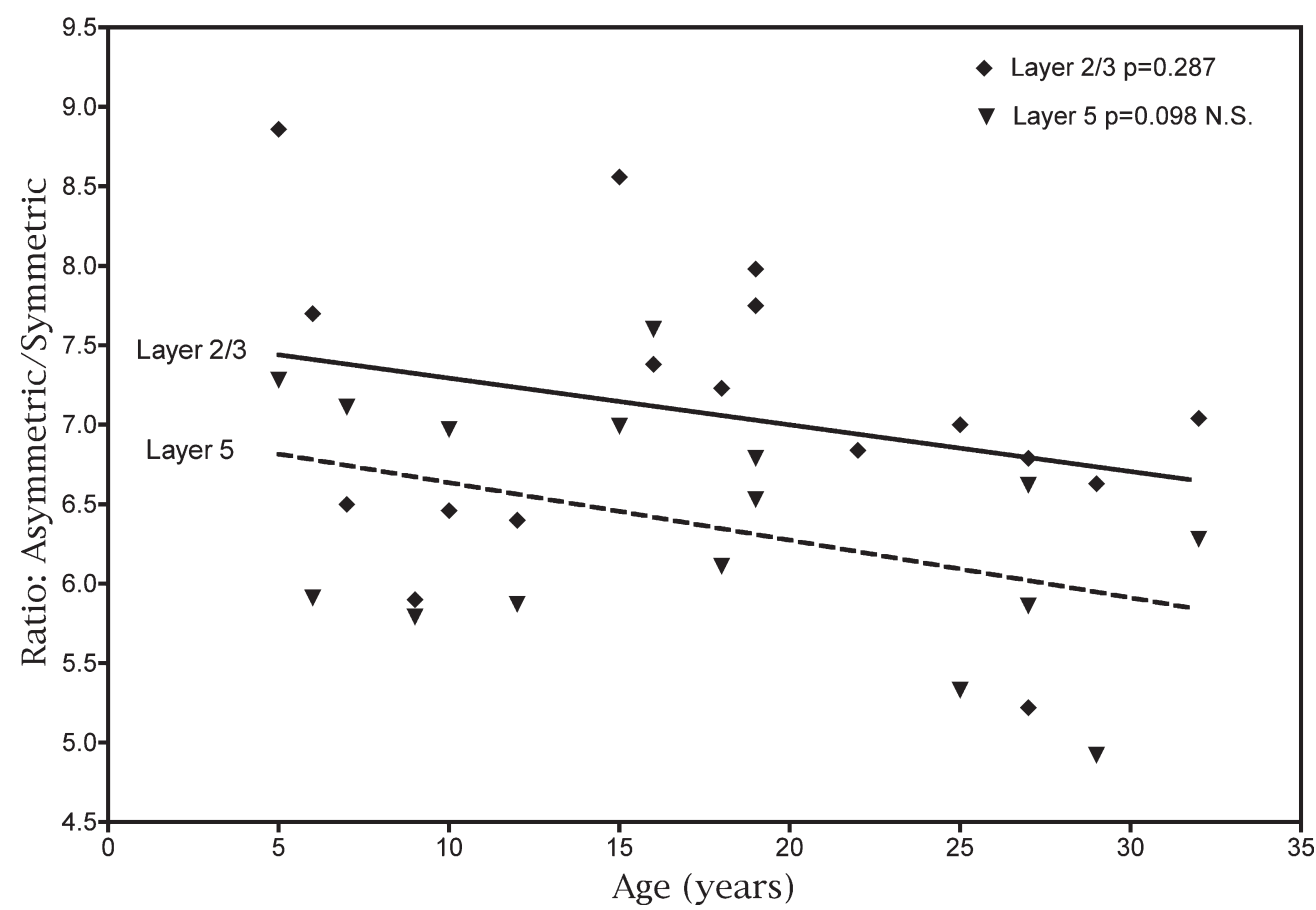

Fig. 4. A plot of the ratio of the numerical density of asymmetric to symmetric synapses in layers $2 / 3$ and in layer 5 against age. 
Table 4. Behavioral data

\begin{tabular}{|c|c|c|c|c|c|}
\hline Animal AM no. & Age (y) & Cll & DNMS (errors) & DNMS, 120-min delay & DRST (spatial) \\
\hline 16 & 5 & no data & & & \\
\hline 76 & 6 & 0.08 & 58.0 & 0.91 & 2.35 \\
\hline 129 & 7 & 1.87 & 114.0 & 0.75 & 2.24 \\
\hline 47 & 9 & 0.51 & 21.0 & 0.95 & 2.23 \\
\hline 53 & 10 & 0.32 & 71.0 & 0.93 & 2.06 \\
\hline 42 & 12 & 0.95 & 40.0 & 0.83 & no data \\
\hline 144 & 15 & 0.42 & 42.0 & 0.88 & 1.94 \\
\hline 143 & 16 & 0.00 & 36.0 & 0.86 & 2.56 \\
\hline 221 & 18 & 2.71 & 200.0 & 0.73 & 2.57 \\
\hline 209 & 19 & 0.80 & 52.0 & 0.79 & 2.5 \\
\hline 133 & 19 & 2.46 & 189.0 & 0.82 & 1.96 \\
\hline 178 & 22 & 6.20 & 472.0 & 0.71 & 2.21 \\
\hline 19 & 25 & 1.98 & 111.0 & 0.72 & 2.34 \\
\hline 12 & 27 & 3.31 & 235.0 & 0.77 & 1.97 \\
\hline 62 & 27 & 3.81 & 353.0 & 0.90 & 2.01 \\
\hline 26 & 29 & 1.05 & 83.0 & 0.85 & 1.98 \\
\hline 41 & 32 & 4.51 & 341.0 & 0.74 & 2.22 \\
\hline
\end{tabular}

DNMS basic, total number of errors; DNMS delay, percentage correct; DRST, average span.

As shown in Fig. 3, part of this difference may be attributed to the fact that while an overall linear analysis shows that there is a significant correlation between age and the decreasing $\mathrm{N}_{\mathrm{V}}$ for asymmetric synapses in layer 5 $(P=0.0178)$, a piecewise analysis of the data (Fig. 3; dashed line) shows that the loss of asymmetric synapses with age is gradual. In contrast there does not appear to be a significant loss of symmetric synapses from layer 5 (Fig. 3; $P=0.307)$. In part this accounts for the fact that the loss of synapses from layer 5 with age is less than the loss from layers $2 / 3$, and it also means that the ratio of asymmetric to symmetric synapses in layer 5 tends to decrease with age (Fig. $4 ; P=0.098$ ).

\section{Behavioral correlates}

The scores that the monkeys in this study achieved on the behavioral tasks are given in Table 4.

For layers 2/3, as shown in Fig. 5, there is a strong correlation between the numerical density of asymmetric

Layer 2/3: Nv v. CII

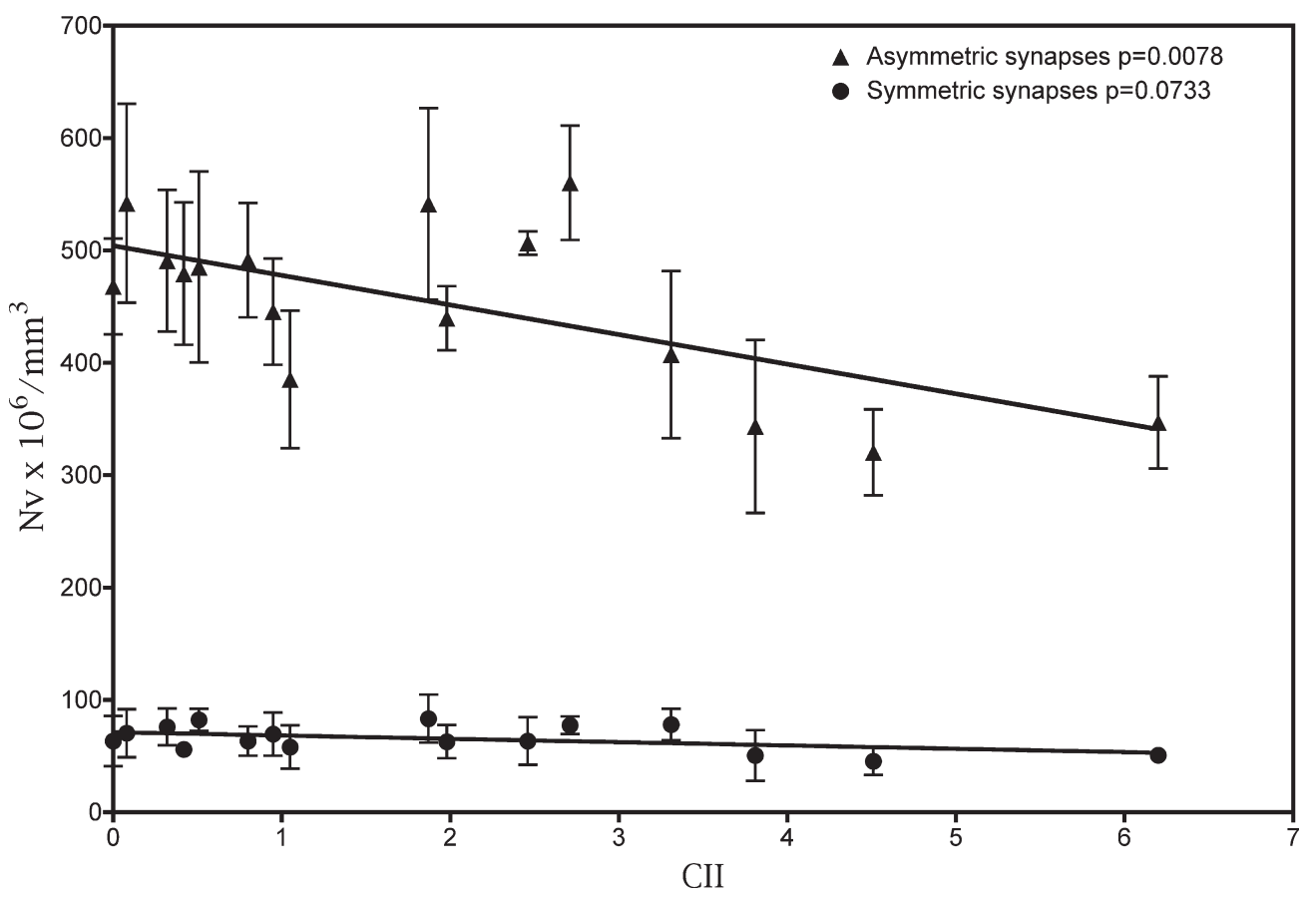

Fig. 5. A plot of the numerical densities of both asymmetric and symmetric synapses in the neuropil of layers $2 / 3$ against the $\mathrm{CII}$ scores attained by the monkeys used in this study. 


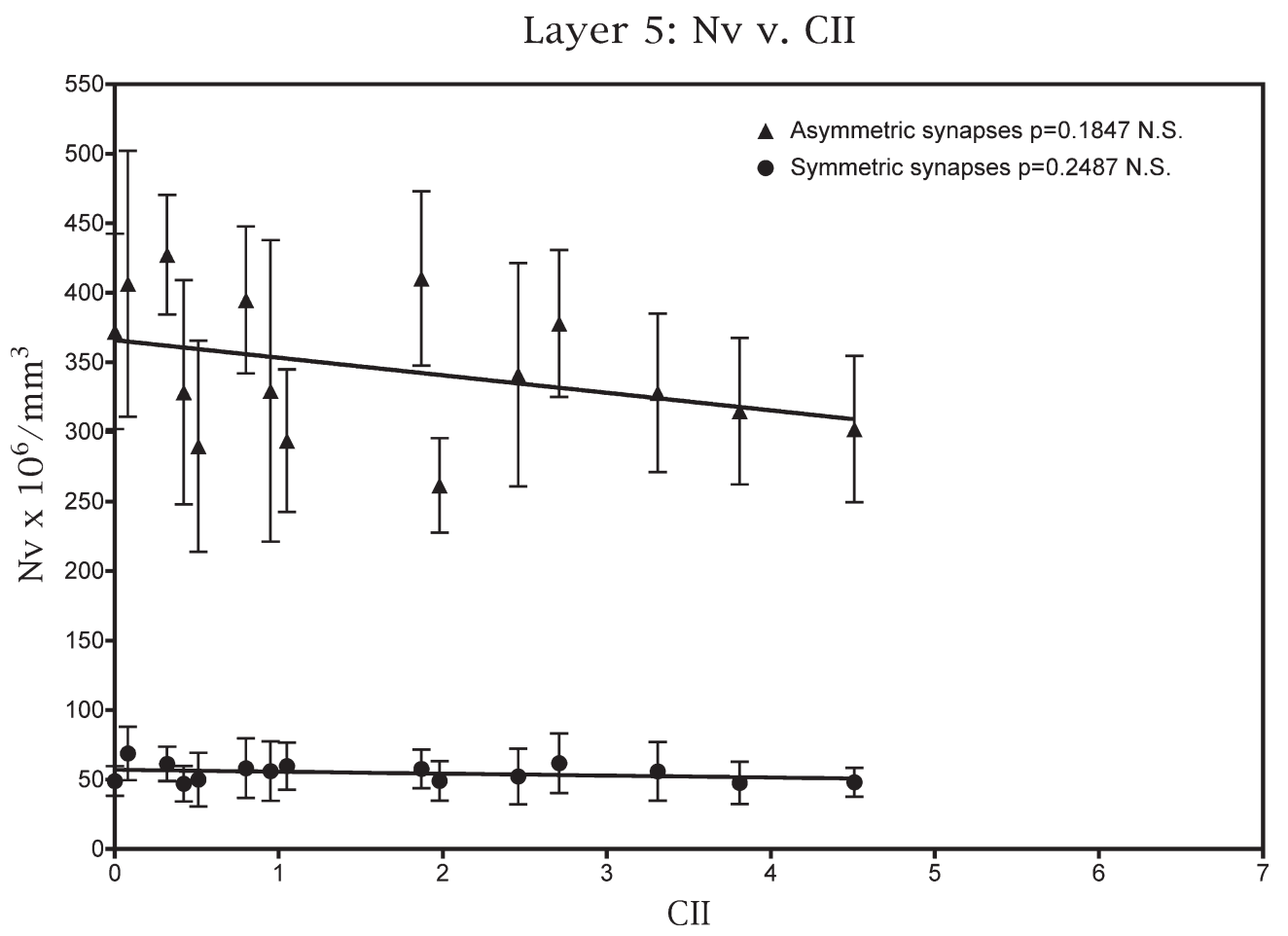

Fig. 6. A plot of the numerical densities of both asymmetric and symmetric synapses in layer 5 against the Cll scores obtained by the monkeys used in this study.

synapses and CII $(P=0.0078)$, but a weaker correlation between the numerical density of symmetric synapses and CII $(P=0.0733)$. In terms of the individual behavioral tasks, there is a strong correlation between the numerical density of asymmetric synapses and the scores on the DNMS basic task $(P=0.006)$ and a weaker correlation between the DNMS scores and the numerical density of symmetric synapses $(P=0.038)$. There is no correlation between the numerical density of synapses and the scores of the monkeys on the DNMS task with a 120-min delay, or on the DRST task.

For layer 5 the results are different. As shown in Fig. 6 , there is no correlation between CII scores and the numerical densities of either the asymmetric $(P=0.248)$ or the symmetric synapses $(P=0.185)$. In addition there are no correlations between numerical densities of asymmetric or symmetric synapses and any of the three individual behavioral tasks.

\section{DISCUSSION}

In summary, this analysis shows that even though there is no loss of neurons, there is a significant overall loss of synapses from layers $2 / 3$ and 5 of prefrontal area 46 and this correlates with age. However, the synaptic losses in the two layers are different. Overall, in layers $2 / 3$ asymmetric and symmetric synapses are lost at equal rates, although the piecewise analysis indicates that the greatest loss of asymmetric synapses occurs after 20 years of age. In layer 5, on the other hand, although there is a loss of asymmetric synapses with age, there appears to be no concomitant loss of symmetric synapses. These differences in synaptic losses from the two layers are also reflected in the correlations between synaptic numerical densities and behavioral measures. Thus for layers $2 / 3$ there is a strong correlation between the numerical density of asymmetric synapses and both the Cll and the basic DNMS task, but a weaker correlation between the numerical density of symmetric synapses and the scores on these two behavioral measures. In contrast, for layer 5 there are no correlations between the numerical densities of synapses and any of the behavioral scores.

However, loss of synapses is not the sole explanation for cognitive decline with age, because we have shown that the frequency of age-related alterations in myelin sheaths within area 46 also significantly correlates with impairments in cognition (Peters and Sethares, 2002b). It may be important to note that it is not necessarily surprising that synapse loss in layers $2 / 3$, but not in layer 5 , correlates with cognitive performance, because it is the layer $2 / 3$ cortico-cortical pyramidal cells, and not the layer 5 subcortical projecting cells, that are thought to play a key role in the performance of cognitive tasks mediated by the prefrontal cortex.

The overall loss of synapses from layers $2 / 3$ is about $30 \%$ and for layer 5 it is only $20 \%$. However, the loss of synapses with age from layer I of area 46 is even greater, being between 30-60\% (Peters et al., 1998b), because layer 1 is much more affected by aging than deeper layers of cortex. Layer 1 becomes thinner with age and there is a dramatic loss of many of the dendritic branches of the tufts 
of apical dendrites that occupy this layer: and unlike the deeper cortical layers, the numerical densities of synapses in layer 1 of monkeys over a range of ages correlate with declines in CII, and with the cognitive decline shown in each of the three individual behavioral tasks. Interestingly, in a parallel study of layer 1 in primary visual cortex of rhesus monkey (Peters et al., 2001), it was found that while there is a similar thinning of layer 1 with age, as well as a $30-50 \%$ loss of synapses, the synaptic loss does not correlate with the scores obtained by the monkeys on any of the behavioral tasks. It was suggested that these differences between layer 1 in the two cortical areas are probably related to the fact that prefrontal cortex has a greater role in subserving cognition than does primary visual cortex.

\section{Relationship between synaptic loss and physiological responses of prefrontal neurons}

The present finding of significantly age-related reductions in the number of excitatory and inhibitory synapses in the prefrontal cortex would predict that synaptic transmission within this cortical area is likely to be altered, potentially leading to cognitive dysfunction. In a previous study, our group has employed whole cell patch clamp recordings to examine the frequency of spontaneous excitatory glutamatergic and inhibitory GABAergic postsynaptic currents (PSCs) in layer $2 / 3$ pyramidal cells in in vitro slices prepared from young and aged rhesus monkeys (Luebke et al., 2004). Consistent with the present findings of significantly reduced numbers of asymmetric synapses in layers $2 / 3$, the frequency of spontaneous excitatory PSCs was significantly reduced with age, with mean values of $4.2 \pm 0.3 \mathrm{~Hz}$ in aged neurons, compared with $7.4 \pm 1.0 \mathrm{~Hz}$ in young neurons $(P=0.0021)$. Although these experiments were not performed on the same monkeys that were assessed for synapse loss, the synapse loss in the present report, as well as other studies demonstrating significant spine loss with age (Duan et al., 2003), provides a highly plausible explanation for the finding of significantly reduced synaptic excitation in layers $2 / 3$ of the aged rhesus monkey prefrontal cortex.

Luebke et al. (2004) demonstrated that, by contrast, the frequency of spontaneous inhibitory PSCs is significantly increased with age, with mean values of $15.4 \pm 1.1 \mathrm{~Hz}$ in aged, as compared with $10.2 \pm 1.7 \mathrm{~Hz}$ in young neurons $(P=0.014)$. This finding is less consistent with the demonstrated loss of approximately $20 \%$ of inhibitory (symmetric) synapses from layers $2 / 3$ shown in the present study. However, the reported increase in benzodiazepine receptor density on the aged monkey neocortex (Rosene and Nicholson, 1999) could be postulated to result in increased inhibitory drive in the aged neocortex, even in light of reduced numbers of symmetric synapses. But perhaps the most straightforward explanation for the increase in spontaneous inhibitory PSC frequency is an increase in the action-potential dependent release of GABA from presynaptic interneurons, which overcomes the loss of postsynaptic substrate for GABA.
In preliminary studies of layer 5 pyramidal cells of aged versus young rhesus monkeys, a modest reduction in frequency of excitatory synaptic responses has been observed with age (Luebke, unpublished observations), although inhibitory synaptic responses have not been assessed. However, Wong and coworkers (2000) demonstrated that layer 5 output neurons in the aged rat parietal cortex undergo a loss of dendritic spines and synapses, that is positively correlated with decreased frequency of action-potential independent glutamatergic and GABAergic miniature PSCs. Interestingly, despite the significant decrease in synaptic substrate and in the frequency of miniature PSCs, the frequency of spontaneous (action-potential-dependent plus action potential-independent) PSCs is not reduced. Wong et al. (2000) interpret this finding to imply that there is a compensatory increase in action-potential dependent input to pyramidal neurons in the face of decreased synaptic substrate.

In the present study it was not possible to determine the exact postsynaptic elements of the synapses lost from layers $2 / 3$. For example, the axospinous synapses could be on the apical and basal dendrites of layers $2 / 3$ pyramidal cells, as well as on the apical dendrites of layer 5 or 6 pyramidal cells passing through the layer. Without more specific information, it is difficult to accurately predict the impact this age-related synapse loss might have on the excitatory-inhibitory balance within and between neocortical laminae in prefrontal cortex. This very important issue will be addressed in future studies. Using intracellular filled pyramidal cells in each cortical layer of young and old monkeys, we will assess whether there is a differential, layer specific, synapse and spine loss from these neurons.

\section{Other studies of monkey cortex in aging}

As pointed out, earlier Uemura (1980) also examined the effects of age on the synaptic density of superior frontal cortex in nine rhesus monkeys, and found a loss of both dendritic spines and synapses with age. To determine numerical densities of synapses, Uemura (1980) used ethanolic phosphotungstic acid to label synapses and used an empirical formula proposed by Cragg (1967). The values obtained by Uemura (1980) are $40-60 \%$ greater than the ones we obtained, since Uemura obtained values of about $850 \times 10^{6}$ per $\mathrm{mm}^{3}$ for young monkeys and $700 \times 10^{6}$ per $\mathrm{mm}^{3}$ for old monkeys. But nevertheless Uemura (1980) calculated the loss of synapses with age to be about $20 \%$.

It is interesting to examine our results in the light of those obtained by Duan et al. (2003), who examined the effects of age on the dendritic trees and spines of layer 3 corticocortically projecting neurons in area 46 of monkey prefrontal cortex. They found that the average lengths of dendrites in the apical and basal dendritic arbors of these neurons did not change significantly with age. However, when the total dendritic spine numbers were compared in young and old monkeys they found a $43 \%$ loss of spines from the apical dendritic trees, mainly from proximal dendrites, while the spine loss from basal dendritic trees was $27 \%$ and this occurred primarily on distal branches. These values are somewhat higher than the $30 \%$ loss of syn- 
apses that we found from layers $2 / 3$, but essentially both sets of data are in agreement in showing that with age there is a substantial loss of synapses and of dendritic spines, which are the principal postsynaptic components of cortical synapses in area 46.

As far as we are aware, there have been no other detailed numerical studies of the effects of aging on synaptic populations in prefrontal cortices of monkeys, although it should be mentioned that Tigges et al. (1992) found no loss of the symmetric axosomatic synapses on Betz cells in monkey motor cortex.

\section{Effect of aging on synapses in cerebral cortex of humans}

The results of electron microscopic studies on the effects of aging on the human frontal cortex are conflicting. Thus, in a recent study Scheff et al. (2001) determined the effects of age on the numbers of synapses in the superior frontal gyrus (area 9) of the brains of 37 normal humans 20-89 years of age, using the physical disector. They concluded there is no significant loss of synapses with age from layers 3 and 5 of frontal cortex in normal aging, and this result is in accord with the conclusion reached earlier by Huttenlocher (1979). In contrast, Gibson (1983) reported a significant loss of synapses from human frontal cortex during normal aging.

Others have examined the effects of normal aging on human cortex using antibodies to label presynaptic axon terminals, and their results are also conflicting. Thus, Masliah et al. (1993) reported a significant loss of synapses from frontal cortex in normal aging, and Liu and Brun (1995) and Liu et al. (1996) agreed. But Zahn et al. (1993) reported no age-related change in the density of synapse labeling with age. Why these differences have arisen is not clear, but several reasons have been cited, such as the inclusion of brains from patients with early stages of Alzheimer's disease in the studies, postmortem delay in obtaining tissue, and inappropriate methods used to make the synaptic counts (e.g. see Scheff et al., 2001).

Acknowledgments-Supported by NIHINIA grant PO1 AG 00001. The authors express thanks to Dr. Howard Cabral for advice on the statistical analyses of the data, and for carrying out the piecewise analyses.

\section{REFERENCES}

Adams I (1987) Comparison of synaptic changes in the precentral and postcentral cerebral cortex of aging humans: a quantitative ultrastructural study. Neurobiol Aging 8:203-212.

Calverley RKS, Lewis DA, Jones DG (1988) Estimation of the numerical density of synapses in rat neocortex: comparisons of the 'disector' with an 'unfolding' method. J Neurosci Methods 23: 195-205.

Chang Y-M, Rosene DL, Killiany RJ, Mangiamele LA, Luebke JI (2005) Increased action potential firing rates of layers $2 / 3$ pyramidal cells in the prefrontal cortex are significantly related to cognitive performance in aged monkeys. Cereb Cortex 15:409-418.

Chen KS, Masliah E, Mallory M, Gage FH (1995) Synaptic loss in cognitively impaired aged rats is ameliorated by chronic human growth factor infusion. Neuroscience 68:19-27.
Colonnier M (1968) Synaptic patterns on different cell types in the different laminae of the cat visual cortex. Brain Res 9:268-287.

Colonnier M, Beaulieu C (1985) An empirical assessment of stereological formulae applied to the counting of synaptic discs in the cerebral cortex. J Comp Neurol 231:175-179.

Cragg BG (1967) The density of synapses and neurones in the motor and visual areas of the cerebral cortex. J Anat (Lond) 101: 639-654.

DeFelipe J, Marco P, Busturia I, Merchán-Pérez A (1999) Estimation of the number of synapses in cerebral cortex: methodological considerations. Cereb Cortex 9:722-732.

Duan H, Wearne SL, Rocher AB, Macedo A, Morrison JH, Hof PR (2003) Age-related dendritic and spine changes in corticocortically projecting neurons in macaque monkeys. Cereb Cortex 19: 950-961.

Fuster JM (1997) The prefrontal cortex. 3rd ed. New York: LippincotRaven.

Gallagher M, Rapp PR (1997) The use of animal models to study the effects of aging on cognition. Annu Rev Psychol 48:339-370.

Gibson PH (1983) EM study of the numbers of cortical synapses in the brains of ageing people and people with Alzheimer-type dementia. Acta Neuropathol (Berl) 62:127-133.

Herndon J, Moss MB, Killiany RJ, Rosene DL (1997) Patterns of cognitive decline in early, advanced and oldest of the old aged rhesus monkeys. Behav Res 87:25-34.

Hof PR, Nimchinsky EA, Young WG, Morrison JH (2000) Numbers of Meynert and layer IVB cells in area V1: a stereologic analysis in young and aged macaque monkeys. J Comp Neurol 420:113-126.

Huttenlocher PR (1979) Synaptic density in human frontal cortexdevelopmental changes and the effects of aging. Brain Res 163:195-205.

Jacobs B, Driscoll I, Schall M (1997) Age related dendritic and spine changes in area 10 and 18 of human cortex: a quantitative Golgi study. J Comp Neurol 386:661-680.

Killiany RJ, Moss MB, Rosene DL, Herndon J (2000) Recognition memory function in early senescent rhesus monkeys. Psychobiology $28: 45-56$.

Kojima S, Goldman-Rakic P (1982) Delay-related activity of prefrontal neurons in rhesus monkeys performing delayed response tasks. Brain Res 248:43-49.

Lai Z, Moss MB, Rosene DL, Herndon J, Killiany R (1995) Executive system dysfunction in aged monkeys: spatial and object reversal learning. Neurobiol Aging 16:947-954.

Liu X, Brun A (1995) Synaptophysin immunoreactivity is stable $36 \mathrm{~h}$ postmortem. Dementia 6:211-217.

Liu X, Erikson C, Brun A (1996) Cortical synaptic changes and gliosis in normal aging, Alzheimer's disease and frontal lobe degeneration. Dementia 7:128-134.

Luebke Jl, Chang Y-M, Moore TL, Rosene DL (2004) Normal aging results in decreased synaptic excitation and increased synaptic inhibition of layers $2 / 3$ pyramidal cells in the monkey prefrontal cortex. Neurosci 125:277-288.

Masliah E, Mallory M, Hansen L, DeTeresa R, Terry RD (1993) Quantitative synaptic alterations in the human neocortex during normal aging. Neurology 43:192-197.

Moore TL, Killiany RJ, Herndon JG, Rosene DL, Moss MB (2003) Impairment in abstraction and set shifting in aged rhesus monkeys. Neurobiol Aging 24:125-134.

Moss MB, Killiany RJ, Herndon JG (1999) Age-related cognitive decline in rhesus monkey. In: Neurodegenerative and age-related changes in structure and function of the cerebral cortex. Cerebral Cortex, Vol. 14 (Peters A, Morrison JH, eds), pp 21-48. New York: Kluwer Academic/Plenum Publishers.

Moss MB, Killiany RJ, Lai C, Rosene DL, Herndon JG (1997) Recognition memory span in rhesus monkeys of advanced age. Neurobiol Aging 18:13-19.

Moss MB, Moore TL, Schettler SP, Killiany R, Rosene DL (2007) Successful $v$ unsuccessful aging in the rhesus monkey. In: Brain 
aging. Models, methods and mechanisms (Riddle DR, ed), pp 21-38. Baton Rouge: CRC Press.

O'Donnell KA, Rapp PR, Hof PR (1999) Preservation of cortical volume in behaviorally characterized aged macaque monkeys. Exp Neurol 160:300-310.

Peters A (2002) Structural changes in the normally aging cerebral cortex of primates. Prog Brain Res 136:455-465.

Peters A, Kaiserman-Abramof IR (1969) The small pyramidal neuron of the rat cerebral cortex. The synapses on dendritic spines. $Z$ Zellforsch 100:487-506.

Peters A, Leahu D, Moss MB, McNally KJ (1994) The effects of aging on area 46 of the frontal cortex of the rhesus monkey. Cereb Cortex 4:621-635.

Peters A, Morrison JH, Rosene DL, Hyman BT (1998a) Are neurons lost from the primate cerebral cortex during normal aging? Cereb Cortex 8:295-300.

Peters A, Sethares C, Moss MB (1998b) The effects of aging on layer 1 in area 46 of prefrontal cortex in the rhesus monkey. Cereb Cortex 8:671-684.

Peters A, Moss MB, Sethares C (2000) Effects of aging on myelinated nerve fibers in monkey primary visual cortex. J Comp Neurol 419:364-376.

Peters A, Moss MB, Sethares C (2001) The effects of aging on layer 1 of primary visual cortex in the rhesus monkey. Cereb Cortex 11:93-103.

Peters A, Palay SL (1996) The morphology of synapses. J Neurocytol 25:687-700.

Peters A, Palay SL, de Webster HF (1991) The fine structure of the nervous system. Neurons and their supporting cells. New York: Oxford University Press.

Peters A, Rosene DL, Moss MB, Kemper TL, Abraham CR, Tigges J, Albert MS (1996) Neurological bases of age-related cognitive de- cline in the rhesus monkey. J Neuropathol Exp Neurol 55: 861-874.

Peters A, Sethares C (2002a) The effects of age on the cells in layer 1 of primate cerebral cortex. Cereb Cortex 12:27-36.

Peters A, Sethares C (2002b) Aging and the myelinated fibers in prefrontal cortex and corpus callosum of the monkey. $\mathrm{J}$ Comp Neurol 442:277-291.

Rosene DL, Nicholson TJ (1999) Some neurotransmitter receptor changes in the hippocampus and cerebral cortex in normal aging. In: Cerebral cortex Vol. 14. Neurodegenerative and age-related changes in cerebral cortex (Peters A, Morrison JH, eds), pp 111128. New York: Kluwer Academic/Plenum Publishers.

Scheff SW, Price DA, Sparks LD (2001) Quantitative assessment of possible age-related change in synaptic numbers in the human frontal cortex. Neurobiol Aging 22:355-365.

Sterio D (1984) The unbiased estimation of number and size of arbitrary particles using the disector. J Microsc 134:127-136.

Tigges J, Herndon JG, Peters A (1992) Axon terminals on Betz cell somata of area 4 in rhesus monkey throughout adulthood. Anat $\operatorname{Rec} 232: 305-315$.

Uemura E (1980) Age-related changes in prefrontal cortex of Macaca mulatta: synaptic density. Exp Neurol 69:164-172.

Walker AE (1940) A cytoarchitectural study of the prefrontal area of the macaque monkey. J Comp Neurol 73:59-86.

Wong TP, Marchese G, Casu MA, Ribeiro-da-Silva A, Cuello AC, De Koninck Y (2000) Loss of presynaptic and postsynaptic structures is accompanied by compensatory increase in action potentialdependent synaptic input to layer $\mathrm{V}$ neocortical pyramidal neurons in aged rats. J Neurosci 20:8596-8606.

Zahn SS, Beyreuther K, Schmitt HP (1993) Quantitative assessment of the synaptophysin immuno-reactivity of the cortical neuropil in various neurodegenerative disorders with dementia. Dementia 4:66-74 